\title{
Analysis of Supply Chain Super-Network Coordination Considering Risk Function in Emergencies
}

\author{
Yafei Zhao \\ School of Chongqing University of Posts and Telecommunications, Chongqing, 400065, China
}

\begin{abstract}
Economic globalization continues to expand the scope of the supply chain network structure, while increasing its own complexity, as well as the uncertainty of the network operating environment and the fragility of the operating system. An emergency on a single node or line in the supply chain network usually affects other nodes in the supply chain and brings significant risks to the enterprise. The impact of other nodes can cause the entire supply chain network to collapse, especially if the production and operation of a single-node enterprise in the supply chain may be interrupted or malfunctioned, especially in the event of an emergency. It also threatens development greatly, affecting the production and livelihoods of enterprises in the supply chain and people's lives, and has a major negative impact on social and economic development. These emergencies continue to affect the supply chain network, and the originally fragile companies face greater risks. This paper establishes a supply chain hyper-network model considering the risk function under emergencies. When an emergency occurs, the demand in the consumer market decreases or increases due to different emergencies. Therefore, revenue sharing contracts are used to coordinate, build a supply chain network model under emergencies, and solve them to obtain a model equilibrium Solution, that is, the new equilibrium state after the occurrence of an emergency.
\end{abstract}

Keywords: Supply chain network, Risk, Supply chain coordination.

\section{Introduction}

Economic globalization has made the scope of the supply chain network structure continue to expand, while increasing its own complexity, but also increasing the uncertainty of the network operation environment and the fragility of the operation system. Sudden events in a single node or line of the supply chain network often affect each other node in the supply chain, which brings great risks to the enterprise. Especially under the risk of emergencies, if the production and operation of a single node enterprise in the supply chain may be interrupted or fail, it may cause the collapse of the entire supply chain network, which greatly threatens the survival and development of other node enterprises in the supply chain, and even affects people's production and life. And social and economic development has brought about a major negative impact. These emergencies continue to impact the supply chain network, making the originally fragile companies face greater risks.

As the types of emergencies increase, the impact increases, and the complexity of events increases, the existing research is relatively insufficient to deal with the impact of emergencies on the supply chain. How to improve the overall resistance of the supply chain and enhance the coordination ability of the supply chain is particularly important important.

Appropriate contracts can effectively coordinate and control the relationship between members in the supply chain and reduce the cost of channel conflicts. Commonly used coordination contracts mainly include: wholesale price, repurchase, revenue sharing, options, and two pricing contracts, etc. Ma Chao [1] considers a two-level supply chain with a loss-averse retailer and a risk-neutral supplier. Luo Xinxing[2] studied the contract coordination problem of the supply chain with the sales promotion changes. Li Feng[3] expanded from a four-level coal supply chain composed of a single supplier to a four-level coal supply chain composed of multiple suppliers, using revenue sharing contracts to study the distribution of benefits in the coal supply chain under random demand. Li Yuan [4] studied the coordination problem of a single-cycle two-level supply chain under the consignment contract with revenue sharing. Wei Guangxing [5]studied the general situation of random market demand and introduced the fairness preference of retailers and suppliers at the same time, using Nash bargaining as the game framework to study the influence of fairness preference on the coordination of revenue sharing contracts. Sun Yuling [6] studied the coordination of the supply chain of fresh agricultural products when supply chain members have fair concerns under the revenue sharing contract.

To sum up, the development of supply chain networks is affected by various emergencies. Research on the balance and coordination of supply chain networks will effectively solve the problems faced by various nodes caused by emergencies and improve the overall risk response capabilities of the supply chain. Reduce the impact of emergencies on the supply chain of various industries.

\section{Model Building}

\subsection{The Purpose of Supply Chain Network Coordination in Emergencies}

The occurrence of emergencies not only affects one node in the supply chain, but also affects other nodes in the supply chain. When emergencies break the original supply chain balance of the supply chain, the revenue sharing contract is adopted as the coordination method, Make the supply chain reach a new equilibrium relatively quickly, and maximize the profit of the supply chain.

Revenue sharing contract: The usual revenue sharing contract is that the retailer shares part of the revenue. It is also assumed that all the profits of the retailer are shared. First find the retailer's total profit, and then distribute it to raw material suppliers, manufacturers, and retailers in a certain proportion.

The retailer's profit maximization is expressed as follows: 


$$
\begin{gathered}
\operatorname{Max} Z_{k}-\alpha_{k} R_{k}=\sum_{j=1}^{J} p_{k j} q_{k j}-\sum_{n=1}^{N} p_{n k} q_{n k}-\sum_{n=1}^{N} c_{3 n k}\left(q_{n k}, h_{n k}\right)-\sum_{j=1}^{J} c_{k j}\left(q_{k j}\right)- \\
\sum_{n=1}^{N} v_{3 n k}\left(k_{n k}\right)-\alpha_{k} \sum_{n=1}^{N} r_{3 n k}\left(q_{n k}, h_{n k}\right)
\end{gathered}
$$

\subsection{Model Symbol Description}

In order to concisely describe each level of decisionmakers and their goals, replace the numbers involved in the decision-making process with the following symbols:

$M$ : The total number of raw material suppliers,

$N$ : Total number of manufacturers: Total number of retailers,

$K:$ The total number of consumers,

$J$ : The number of raw materials sold by the supplier of raw materials to the manufacturer after the emergency,

$\tilde{q}_{m n}$ : the number of products sold by the manufacturer to the retailer after the emergency,

$\tilde{q}_{n k}$ : The number of retailers sold to consumers after the emergency,

$\tilde{p}_{m n}$ : The price that the raw material supplier sells to the manufacturer after the emergency,

$\tilde{p}_{n k}$ : The price at which the manufacturer sells the product to the retailer after the emergency,
$\tilde{P}_{k j}:$ The price at which retailers sell products to consumers after an emergency,

$\tilde{h}_{m n}$ : The level of the relationship between the raw material supplier and the manufacturer after the emergency occurs,

$\tilde{h}_{n k}$ : The relationship level of the manufacturer-retailer after the incident occurred

\subsection{Behavioral Decision-making and Target Analysis at All Levels}

\subsubsection{Raw Material Supplier Behavior and Target}

\section{Analysis}

Let $\omega$ denote the proportion of the profit held by the raw material supplier $m$ in the profit on the supply chain, let $\mu$ denote the proportion of the profit held by the manufacturer $n$ in the profit on the supply chain, then the proportion of the profit held by the retailer $k$ is $1-\mu-\omega . \mu$ and $\omega$ represent constant values, which are the result of coordination among various companies in the supply chain. By changing the size of $\mu$ and $\omega$, the profit distribution of each enterprise in the supply chain can be adjusted.the profit of manufacturer $m$ can be expressed as:

$$
\begin{aligned}
\operatorname{Max} U_{m}= & \omega\left(\sum_{j=1}^{J} \tilde{p}_{k j} \tilde{q}_{k j}-\sum_{n=1}^{N} \tilde{p}_{n k} \tilde{q}_{n k}-\sum_{n=1}^{N} c_{3 n k}\left(\tilde{q}_{n k}, \tilde{h}_{n k}\right)-\sum_{j=1}^{J} c_{k j}\left(\tilde{q}_{k j}\right)-\right. \\
& \left.\sum_{n=1}^{N} v_{3 n k}\left(\tilde{k}_{n k}\right)-\alpha_{k} \sum_{n=1}^{N} r_{3 n k}\left(\tilde{q}_{n k}, \tilde{h}_{n k}\right)\right)-f_{m}\left(\tilde{Q}_{1}\right)-\sum_{n=1}^{N} c_{1 m n}\left(\tilde{q}_{m n}, \tilde{h}_{m n}\right) \\
& -\sum_{n=1}^{N} \tilde{v}_{1 m n}-\alpha_{m} \sum_{n=1}^{N} r_{m n}\left(\tilde{q}_{m n}, \tilde{h}_{m n}\right)
\end{aligned}
$$

According to the theory of variational inequality, (2) can be

transformed into the following variational inequality:

$$
\begin{aligned}
& \sum_{m=1}^{M} \sum_{n=1}^{N}\left(\frac{\partial f_{m}\left(\tilde{Q}_{1}^{*}\right)}{\partial \tilde{q}_{m n}}+\frac{\partial c_{1 m n}\left(\tilde{q}_{m n}^{*}, \tilde{h}_{m n}^{*}\right)}{\partial \tilde{q}_{m n}}-\tilde{p}_{m n}^{*}+\alpha_{m} \frac{\partial r_{m n}\left(\tilde{q}_{m n}^{*}, \tilde{h}_{m n}^{*}\right)}{\partial \tilde{q}_{m n}}\right) \times\left(\tilde{q}_{m n}-\tilde{q}_{m n}^{*}\right)+ \\
& \sum_{m=1}^{M} \sum_{n=1}^{N}\left(\frac{\partial c_{1 m n}\left(\tilde{q}_{m n}^{*}, \tilde{h}_{m n}^{*}\right)}{\partial \tilde{h}_{m n}}-\frac{\partial v_{1 m n}\left(\tilde{h}_{m n}^{*}\right)}{\partial \tilde{h}_{m n}}+\alpha_{m} \frac{\partial r_{m n}\left(\tilde{q}_{m n}^{*}, \tilde{h}_{m n}^{*}\right)}{\partial \tilde{h}_{m n}}\right) \times\left(\tilde{h}_{m n}-\tilde{h}_{m n}^{*}\right) \geqslant 0
\end{aligned}
$$




\subsubsection{Manufacturer's Behavior and Target Analysis}

$$
\begin{aligned}
\operatorname{Max} U_{n}= & \mu\left(\sum_{j=1}^{J} \tilde{p}_{k j} \tilde{q}_{k j}-\sum_{n=1}^{N} \tilde{p}_{n k} \tilde{q}_{n k}-\sum_{n=1}^{N} c_{3 n k}\left(\tilde{q}_{n k}, \tilde{h}_{n k}\right)-\sum_{j=1}^{J} c_{k j}\left(\tilde{q}_{k j}\right)-\right. \\
& \left.\sum_{n=1}^{N} v_{3 n k}\left(\tilde{k}_{n k}\right)-\alpha_{k} \sum_{n=1}^{N} r_{3 n k}\left(\tilde{q}_{n k}, \tilde{h}_{n k}\right)\right)-\sum_{k=1}^{K} \tilde{p}_{n k} \tilde{q}_{n k}-\sum_{k=1}^{K} c_{2 n k}\left(\tilde{q}_{n k}, \tilde{h}_{n k}\right)- \\
& \sum_{m=1}^{M} \tilde{p}_{m n} \tilde{q}_{m n}-\sum_{m=1}^{M} c_{2 m n}\left(\tilde{q}_{m n}, \tilde{h}_{m n}\right)-\alpha_{n}\left(\sum_{m=1}^{M} r_{2 m n}\left(\tilde{q}_{m n}, \tilde{h}_{m n}\right)+\sum_{k=1}^{K} r_{2 n k}\left(\tilde{q}_{n k}, \tilde{h}_{n k}\right)\right) \\
& -\sum_{m=1}^{M} v_{2 m n}\left(\tilde{h}_{m n}\right)-\sum_{k=1}^{K} v_{2 n k}\left(\tilde{h}_{n k}\right)
\end{aligned}
$$

According to the theory of variational inequality, (4) can be transformed into the following variational inequality:

$$
\begin{aligned}
& \sum_{m=1}^{M} \sum_{n=1}^{N}\left(\frac{\partial c_{2 m n}\left(\tilde{q}_{m n}^{*}, \tilde{h}_{m n}^{*}\right)}{\partial q_{m n}}+\frac{\partial r_{2 m n}\left(\tilde{q}_{m n}^{*}, \tilde{h}_{m n}^{*}\right)}{\partial q_{m n}}+\tilde{p}_{m n}^{*}-\omega \zeta_{1 n}^{*}\right) \times\left(\tilde{q}_{m n}-\tilde{q}_{m n}^{*}\right)+ \\
& \sum_{m=1}^{M} \sum_{n=1}^{N}\left(\frac{\partial c_{2 m n}\left(\tilde{q}_{m n}^{*}, \tilde{h}_{m n}^{*}\right)}{\partial h_{m n}}+\frac{\partial r_{2 m n}\left(\tilde{q}_{m n}^{*}, \tilde{h}_{m n}^{*}\right)}{\partial h_{m n}}+\frac{\partial v_{2 m n}\left(\tilde{h}_{m n}^{*}\right)}{\partial h_{m n}}\right) \times\left(\tilde{h}_{m n}-\tilde{h}_{m n}^{*}\right)+ \\
& \sum_{n=1}^{N} \sum_{k=1}^{K}\left(\frac{\partial c_{2 n k}\left(\tilde{q}_{n k}^{*}, \tilde{h}_{n k}^{*}\right)}{\partial \tilde{q}_{n k}}+\frac{\partial r_{2 n k}\left(\tilde{q}_{n k}^{*}, \tilde{h}_{n k}^{*}\right)}{\partial \tilde{q}_{n k}}-\tilde{p}_{n k}^{*}+\omega \zeta_{1 n}^{*}\right) \times\left(\tilde{q}_{n k}-\tilde{q}_{n k}^{*}\right)+ \\
& \sum_{n=1}^{N} \sum_{k=1}^{K}\left(\frac{\partial c_{2 n k}\left(\tilde{q}_{n k}^{*}, \tilde{h}_{n k}^{*}\right)}{\partial \tilde{h}_{n k}}+\frac{\partial r_{2 n k}\left(\tilde{q}_{n k}^{*}, \tilde{h}_{n k}^{*}\right)}{\partial \tilde{h}_{n k}}-\frac{\partial v_{2 n k}\left(\tilde{h}_{n k}\right)}{\partial \tilde{h}_{n k}}\right) \times\left(\tilde{h}_{n k}-\tilde{h}_{n k}^{*}\right)+ \\
& \sum_{n=1}^{N}\left(\sum_{m=1}^{M} \tilde{q}_{m n}^{*}-\sum_{k=1}^{K} \tilde{q}_{n k}^{*}\right) \times\left(\zeta_{1 n}-\zeta_{1 n}^{*}\right) \geqslant 0
\end{aligned}
$$

\subsubsection{Retailer's Behavior and Target Analysis}

of retailer $k$ is $(1-\mu-\omega)$, and the specific function is as follows:

Similarly, according to equation (1), the profit percentage

$$
\begin{aligned}
\operatorname{Max} U_{k} & =(1-\varepsilon-\mu)\left(\sum_{j=1}^{J} \tilde{p}_{k j} \tilde{q}_{k j}-\sum_{n=1}^{N} \tilde{p}_{n k} \tilde{q}_{n k}-\sum_{n=1}^{N} c_{3 n k}\left(\tilde{q}_{n k}, \tilde{h}_{n k}\right)-\sum_{j=1}^{J} c_{k j}\left(\tilde{q}_{k j}\right)-\right. \\
& \left.\sum_{n=1}^{N} v_{3 n k}\left(\tilde{k}_{n k}\right)-\alpha_{k} \sum_{n=1}^{N} r_{3 n k}\left(\tilde{q}_{n k}, \tilde{h}_{n k}\right)\right)-\sum_{j=1}^{J} \tilde{p}_{k j} \tilde{q}_{k j}-\sum_{n=1}^{N} \tilde{p}_{n k} \tilde{q}_{n k}-\sum_{n=1}^{N} c_{3 n k}\left(\tilde{q}_{n k}, \tilde{h}_{n k}\right) \\
& -\sum_{j=1}^{J} c_{k j}\left(\tilde{q}_{k j}\right)-\sum_{n=1}^{N} v_{3 n k}\left(\tilde{k}_{n k}\right)-\alpha_{k} \sum_{n=1}^{N} r_{3 n k}\left(\tilde{q}_{n k}, \tilde{h}_{n k}\right)
\end{aligned}
$$

According to the theory of variational inequality, (6) can be transformed into the following variational inequality: 


$$
\begin{aligned}
& \sum_{n=1}^{N} \sum_{k=1}^{K}\left(\frac{\partial c_{3 n k}\left(\tilde{q}_{n k}^{*}, \tilde{h}_{n k}^{*}\right)}{\partial \tilde{q}_{n k}}+\alpha_{k} \frac{\partial r_{3 n k}\left(\tilde{q}_{n k}^{*}, \tilde{h}_{n k}^{*}\right)}{\partial \tilde{q}_{n k}}+\tilde{p}_{n k}^{*}-\mu \zeta_{2 k}^{*}\right) \times\left(\tilde{q}_{n k}-\tilde{q}_{n k}^{*}\right)+ \\
& \sum_{n=1}^{N} \sum_{k=1}^{K}\left(\frac{\partial c_{3 n k}\left(\tilde{q}_{n k}^{*}, \tilde{h}_{n k}^{*}\right)}{\partial \tilde{h}_{n k}}+\alpha_{k} \frac{\partial r_{3 n k}\left(\tilde{q}_{n k}^{*}, \tilde{h}_{n k}^{*}\right)}{\partial \tilde{h}_{n k}}+\frac{\partial v_{3 n k}\left(\tilde{k}_{n k}^{*}\right)}{\partial \tilde{h}_{n k}}\right) \times\left(\tilde{h}_{n k}-\tilde{h}_{n k}^{*}\right)+ \\
& \sum_{k=1}^{K} \sum_{j=1}^{J}\left(\frac{\partial c_{k j}\left(\tilde{q}_{k j}^{*}\right)}{\partial \tilde{q}_{k j}}-\tilde{p}_{k j}^{*}+\mu \zeta_{2 k}^{*}\right) \times\left(\tilde{q}_{k j}-\tilde{q}_{k j}^{*}\right)+\sum_{k=1}^{K}\left(\sum_{n=1}^{N} \tilde{q}_{n k}^{*}-\sum_{j=1}^{J} \tilde{q}_{k j}^{*}\right) \times \\
& \left(\zeta_{2 k}-\zeta_{2 k}^{*}\right)+\geqslant 0
\end{aligned}
$$

\subsubsection{Demand Market Behavior and Target Analysis}

When an emergency occurs, a certain consumer demand is affected, which represents the consumer market's demand function for retailer's products. The demand function of normal goods is a monotonically decreasing function of price.

Let a denote the consumer's satisfaction level after purchasing the product from the retailer. The quantity and price of purchased goods will have a direct impact on consumer utility. bc, where d represents the consumer's sensitivity to the quantity and price of the product, and $\mathrm{e}$ is a non-negative number, which summarizes the consumer's utility to other characteristics of the retailer.

Then the objective function of the consumer is

$$
\operatorname{Max} U_{j}=\sum_{j=1}^{J} u_{k j}\left(\tilde{p}_{k j}, \tilde{q}_{k j}\right)-\sum_{k=1}^{K} \tilde{p}_{k j} \tilde{q}_{k j}-\sum_{k=1}^{K} c_{k j}\left(\tilde{q}_{k j}\right)
$$

$$
\sum_{k=1}^{K} \tilde{q}_{k j}=d_{j}(\tilde{p})
$$

According to the functions (1), (8), (9), the optimal solution is equivalent to the solution of the following variational inequality:

\subsection{Model Dynamic Equilibrium Analysis}

According to the related theorems of variational inequality, the model has a unique solution and satisfies the following conditions:

$$
\begin{aligned}
& \sum_{m=1}^{M} \sum_{n=1}^{N}\left(\frac{\partial f_{m}\left(\tilde{Q}_{1}^{*}\right)}{\partial \tilde{q}_{m n}}+\frac{\partial c_{1 m n}\left(\tilde{q}_{m n}^{*}, \tilde{h}_{m n}^{*}\right)}{\partial \tilde{q}_{m n}}-\tilde{p}_{m n}^{*}+\alpha_{m} \frac{\partial r_{m n}\left(\tilde{q}_{m n}^{*}, \tilde{h}_{m n}^{*}\right)}{\partial \tilde{q}_{m n}}\right) \times\left(\tilde{q}_{m n}-\tilde{q}_{m n}^{*}\right)+ \\
& \sum_{m=1}^{M} \sum_{n=1}^{N}\left(\frac{\partial c_{1 m n}\left(\tilde{q}_{m n}^{*}, \tilde{h}_{m n}^{*}\right)}{\partial \tilde{h}_{m n}}-\frac{\partial v_{1 m n}\left(\tilde{h}_{m n}^{*}\right)}{\partial \tilde{h}_{m n}}+\alpha_{m} \frac{\partial r_{m n}\left(\tilde{q}_{m n}^{*}, \tilde{h}_{m n}^{*}\right)}{\partial \tilde{h}_{m n}}\right) \times\left(\tilde{h}_{m n}-\tilde{h}_{m n}^{*}\right)+ \\
& \sum_{m=1}^{M} \sum_{n=1}^{N}\left(\frac{\partial c_{2 m n}\left(\tilde{q}_{m n}^{*}, \tilde{h}_{m n}^{*}\right)}{\partial q_{m n}}+\frac{\partial r_{2 m n}\left(\tilde{q}_{m n}^{*}, \tilde{h}_{m n}^{*}\right)}{\partial q_{m n}}+\tilde{p}_{m n}^{*}-\omega \zeta_{1 n}^{*}\right) \times\left(\tilde{q}_{m n}-\tilde{q}_{m n}^{*}\right)+ \\
& \sum_{m=1}^{M} \sum_{n=1}^{N}\left(\frac{\partial c_{2 m n}\left(\tilde{q}_{m n}^{*}, \tilde{h}_{m n}^{*}\right)}{\partial h_{m n}}+\frac{\partial r_{2 m n}\left(\tilde{q}_{m n}^{*}, \tilde{h}_{m n}^{*}\right)}{\partial h_{m n}}+\frac{\partial v_{2 m n}\left(\tilde{h}_{m n}^{*}\right)}{\partial h_{m n}}\right) \times\left(\tilde{h}_{m n}-\tilde{h}_{m n}^{*}\right)+ \\
& \sum_{n=1}^{N} \sum_{k=1}^{K}\left(\frac{\partial c_{2 n k}\left(\tilde{q}_{n k}^{*}, \tilde{h}_{n k}^{*}\right)}{\partial \tilde{q}_{n k}}+\frac{\partial r_{2 n k}\left(\tilde{q}_{n k}^{*}, \tilde{h}_{n k}^{*}\right)}{\partial \tilde{q}_{n k}}-\tilde{p}_{n k}^{*}+\omega \zeta_{1 n}^{*}\right) \times\left(\tilde{q}_{n k}-\tilde{q}_{n k}^{*}\right)+ \\
& \sum_{n=1}^{N} \sum_{k=1}^{K}\left(\frac{\partial c_{2 n k}\left(\tilde{q}_{n k}^{*}, \tilde{h}_{n k}^{*}\right)}{\partial \tilde{h}_{n k}}+\frac{\partial r_{2 n k}\left(\tilde{q}_{n k}^{*}, \tilde{h}_{n k}^{*}\right)}{\partial \tilde{h}_{n k}}-\frac{\partial v_{2 n k}\left(\tilde{h}_{n k}\right)}{\left.\partial \tilde{h}_{n k}\right) \times\left(\tilde{h}_{n k}-\tilde{h}_{n k}^{*}\right)+}\right.
\end{aligned}
$$




$$
\begin{aligned}
& \sum_{n=1}^{N}\left(\sum_{m=1}^{M} \tilde{q}_{m n}^{*}-\sum_{k=1}^{K} \tilde{q}_{n k}^{*}\right) \times\left(\zeta_{1 n}-\zeta_{1 n}^{*}\right)+\sum_{k=1}^{K}\left(\sum_{n=1}^{N} \tilde{q}_{n k}^{*}-\sum_{j=1}^{J} \tilde{q}_{k j}^{*}\right) \times\left(\zeta_{2 k}-\zeta_{2 k}^{*}\right)+ \\
& \sum_{n=1}^{N} \sum_{k=1}^{K}\left(\frac{\partial c_{3 n k}\left(\tilde{q}_{n k}^{*}, \tilde{h}_{n k}^{*}\right)}{\partial \tilde{q}_{n k}}+\alpha_{k} \frac{\partial r_{3 n k}\left(\tilde{q}_{n k}^{*}, \tilde{h}_{n k}^{*}\right)}{\partial \tilde{q}_{n k}}+\tilde{p}_{n k}^{*}-\mu \zeta_{2 k}^{*}\right) \times\left(\tilde{q}_{n k}-\tilde{q}_{n k}^{*}\right)+ \\
& \sum_{n=1}^{N} \sum_{k=1}^{K}\left(\frac{\partial c_{3 n k}\left(\tilde{q}_{n k}^{*}, \tilde{h}_{n k}^{*}\right)}{\partial \tilde{h}_{n k}}+\alpha_{k} \frac{\partial r_{3 n k}\left(\tilde{q}_{n k}^{*}, \tilde{h}_{n k}^{*}\right)}{\partial \tilde{h}_{n k}}+\frac{\partial v_{3 n k}\left(\tilde{k}_{n k}^{*}\right)}{\partial \tilde{h}_{n k}}\right) \times\left(\tilde{h}_{n k}-\tilde{h}_{n k}^{*}\right)+ \\
& \sum_{k=1}^{K} \sum_{j=1}^{J}\left(\frac{\partial c_{k j}\left(\tilde{q}_{k j}^{*}\right)}{\partial \tilde{q}_{k j}}-\tilde{p}_{k j}^{*}+\mu \zeta_{2 k}^{*}\right) \times\left(\tilde{q}_{k j}-\tilde{q}_{k j}^{*}\right)+\sum_{j=1}^{J}\left(\sum_{k=1}^{K} \tilde{q}_{k j}^{*}-d_{j}\left(\tilde{p}_{k j}^{*}\right)\right) \times\left(\zeta_{3 j}-\zeta_{3 j}^{*}\right)+ \\
& \sum_{k=1}^{K} \sum_{j=1}^{J}\left(\frac{\partial c_{k j}\left(\tilde{q}_{k j}^{*}\right)}{\partial \tilde{q}_{k j}}+\tilde{p}_{k j}-\frac{\partial u_{k j}\left(\tilde{p}_{k j}^{*}, \tilde{q}_{k j}^{*}\right)}{\partial \tilde{q}_{k j}}-(1-\omega-\mu) \zeta_{3 j}^{*}\right) \times\left(\tilde{q}_{k j}-\tilde{q}_{k j}^{*}\right)+ \\
& \sum_{k=1}^{K} \sum_{j=1}^{J}\left(\tilde{q}_{k j}^{*}+(1-\omega-\mu) \zeta_{3 j}^{*}-\frac{\partial u_{k j}\left(\tilde{p}_{k j}^{*}, \tilde{q}_{k j}^{*}\right)}{\partial \tilde{p}_{k j}}\right) \times\left(\tilde{p}_{k j}-\tilde{p}_{k j}^{*}\right) \geqslant 0
\end{aligned}
$$

The solution of the variational inequality is further calculated by the improved projection gradient algorithm:

$$
\begin{aligned}
& \tilde{q}_{m n}^{T-1}=\max \left(0, \tilde{q}_{m n}^{T-1}-\theta\left(\frac{\partial f_{m}\left(\tilde{Q}_{1}^{T-1}\right)}{\partial q_{m n}}+\frac{\partial c_{1 m n}\left(\tilde{q}_{m n}^{T-1}, \tilde{h}_{m n}^{T-1}\right)}{\partial q_{m n}}+\alpha_{m} \frac{\partial r_{1 m n}\left(\tilde{q}_{m n}^{T-1}, \tilde{h}_{m n}^{T-1}\right)}{\partial q_{m n}}\right.\right. \\
& \left.\left.\frac{\partial c_{2 m n}\left(\tilde{q}_{m n}^{T-1}, \tilde{h}^{T-1}\right)}{\partial q_{m n}}+\alpha_{n} \frac{\partial r_{2 m n}\left(\tilde{q}_{m n}^{T-1}, \tilde{h}_{m n}^{T-1}\right)}{\partial q_{m n}}-\omega \zeta_{1 n}^{T-1}\right)\right) \\
& \tilde{q}_{n k}^{T-1}=\max \left(0, \tilde{q}_{n k}^{T-1}-\theta\left(\frac{\partial c_{2 n k}\left(\tilde{q}_{n k}^{T-1}, \tilde{h}_{n k}^{T-1}\right)}{\partial \tilde{q}_{m n}}+\alpha_{n} \frac{\partial r_{2 n k}\left(\tilde{q}_{n k}^{T-1}, \tilde{h}_{n k}^{T-1}\right)}{\partial \tilde{q}_{m n}}-\mu \zeta_{2 k}^{T-1}\right.\right. \\
& \left.\left.\frac{\partial c_{3 n k}\left(\tilde{q}_{n k}^{T-1}, \tilde{h}_{n k}^{T-1}\right)}{\partial \tilde{q}_{n k}}+\alpha_{k} \frac{\partial r_{3 n k}\left(\tilde{q}_{n k}^{T-1}, \tilde{h}_{n k}^{T-1}\right)}{\partial \tilde{q}_{n k}}\right)\right) \\
& \tilde{q}_{k j}^{T}=\max \left(0, \tilde{q}_{k j}^{T-1}-\theta\left(\frac{\partial f_{k}\left(\tilde{Q}_{3}^{T-1}\right)}{\partial \tilde{q}_{k j}}+\frac{\partial c_{k j}\left(\tilde{q}_{k j}^{T-1}\right)}{\partial \tilde{q}_{k j}}+\frac{\partial \tilde{c}_{k j}\left(\tilde{q}_{k j}^{T-1}\right)}{\partial \tilde{q}_{k j}}-\right.\right. \\
& \left.\left.(1-\omega-\mu) \zeta_{3 j}^{T-1}-\frac{\partial u_{k j}\left(\tilde{p}_{k j}^{T-1}, \tilde{q}_{k j}^{T-1}\right)}{\partial \tilde{q}_{k j}}\right)\right) \\
& \tilde{p}_{k j}^{T}=\max \left(0, \tilde{p}_{k j}^{T-1}-\theta\left(\tilde{q}_{k j}^{T-1}-\frac{\partial u_{k j}\left(\tilde{p}_{k j}^{T-1}, \tilde{q}_{k j}^{T-1}\right)}{\partial \tilde{p}_{k j}}+(1-\omega-\mu) \zeta_{3 j}^{T-1}\right)\right) \\
& \tilde{h}_{m n}^{T}=\min \left(1, \max \left(0, \tilde{h}_{m n}^{T-1}-\theta\left(\frac{\partial c_{1 m n}\left(\tilde{q}_{m n}^{T-1}, \tilde{h}_{m n}^{T-1}\right)}{\partial \tilde{h}_{m n}}+\frac{\partial \tilde{v}_{1 m n}\left(\tilde{h}_{m n}^{T-1}\right)}{\partial \tilde{h}_{m n}}+\right.\right.\right. \\
& \alpha_{m} \frac{\partial r_{1 m n}\left(\tilde{q}_{m n}^{T-1}, \tilde{h}_{m n}^{T-1}\right)}{\partial h_{m n}}+\frac{\partial c_{2 m n}\left(\tilde{q}_{m n}^{T-1}, \tilde{h}^{T-1}\right)}{\partial \tilde{h}_{m n}}+\alpha_{n} \frac{\partial r_{2 m n}\left(\tilde{q}_{m n}^{T-1}, \tilde{h}_{m n}^{T-1}\right)}{\partial \tilde{h}_{m n}} \\
& \left.\left.\left.+\frac{\partial v_{2 m n}\left(\tilde{h}_{m n}^{T-1}\right)}{\partial \tilde{h}_{m n}}\right)\right)\right)
\end{aligned}
$$




$$
\begin{aligned}
\tilde{h}_{n k}^{T}= & \min \left(1, \max \left(0, \tilde{h}_{n k}^{T-1}-\theta\left(\frac{\partial c_{1 n k}\left(\tilde{q}_{n k}^{T-1}, \tilde{h}_{n k}^{T-1}\right)}{\partial \tilde{h}_{n k}}+\frac{\partial v_{1 n k}\left(\tilde{h}_{n k}^{T-1}\right)}{\partial \tilde{h}_{n k}}+\right.\right.\right. \\
& \alpha_{n} \frac{\partial r_{1 n k}\left(\tilde{q}_{n k}^{T-1}, \tilde{h}_{n k}^{T-1}\right)}{\partial \tilde{h}_{n k}}+\frac{\partial c_{2 n k}\left(\tilde{q}_{n k}^{T-1}, \tilde{h}_{n k}^{T-1}\right)}{\partial \tilde{h}_{n k}}+\alpha_{k} \frac{\partial r_{2 n k}\left(\tilde{q}_{n k}^{T-1}, \tilde{h}_{n k}^{T-1}\right)}{\partial \tilde{h}_{n k}} \\
& \left.\left.\left.+\frac{\partial v_{2 n k}\left(h_{n k}^{T-1}\right)}{\partial \tilde{h}_{n k}}\right)\right)\right)
\end{aligned}
$$$$
\zeta_{1 n}^{T}=\max \left(0, \zeta_{1 n}^{T-1}-\theta\left(\sum_{m=1}^{M} \tilde{q}_{m n}^{T-1}-\sum_{k=1}^{K} \tilde{q}_{n k}^{T-1}\right)\right)
$$$$
\zeta_{2 k}^{T}=\max \left(0, \zeta_{2 k}^{T-1}-\theta\left(\sum_{n=1}^{N} \tilde{q}_{n k}^{T-1}-\sum_{j=1}^{J} \tilde{q}_{k j}^{T-1}\right)\right)
$$$$
\tilde{\zeta}_{3 j}^{T}=\max \left(0, \zeta_{3 j}^{T-1}-\theta\left(\sum_{k=1}^{K} \tilde{q}_{k j}^{T-1}-d_{j}\left(\tilde{p}_{k j}^{T-1}\right)\right)\right)
$$

The economic cost function is

$$
\begin{gathered}
v_{1 m n}\left(h_{m n}\right)=1.5 h_{m n}+2, \forall m, n \\
v_{2 m n}\left(h_{m n}\right)=2 h_{m n}+1, \forall m, n \\
v_{2 n k}\left(h_{n k}\right)=h_{n k}+2, \forall n, k \\
v_{3 n k}\left(h_{n k}\right)=h_{n k}+2, \forall n, k
\end{gathered}
$$

The risk function is:

$$
\begin{aligned}
& r_{1 m n}\left(q_{m n}, h_{m n}\right)=q_{m n}^{2}+q_{m n}-h_{m n}, \forall m, n \\
& r_{2 m n}\left(q_{m n}, h_{m n}\right)=q_{m n}^{2}+2 q_{m n}-h_{m n}, \forall m, n \\
& r_{2 n k}\left(q_{n k}, h_{n k}\right)=2 q_{n k}^{2}+q_{n k}-1.5 h_{n k}, \forall n, k \\
& r_{3 n k}\left(q_{n k}, h_{n k}\right)=q_{n k}^{2}+q_{n k}-2 h_{n k}, \forall n, k
\end{aligned}
$$

The consumer utility function is:

$$
\begin{aligned}
& f_{1}\left(Q_{1}\right)=\left(\sum_{n=1}^{2} q_{1 n}\right)^{2}+0.5\left(\sum_{n=1}^{2} q_{2 n}\right)\left(\sum_{n=1}^{2} q_{1 n}\right)+2\left(\sum_{n=1}^{2} q_{1 n}\right) \\
& f_{2}\left(Q_{1}\right)=0.5\left(\sum_{n=1}^{2} q_{2 n}\right)^{2}+\left(\sum_{n=1}^{2} q_{1 n}\right)\left(\sum_{n=1}^{2} q_{1 n}\right)+\left(\sum_{n=1}^{2} q_{2 n}\right)
\end{aligned}
$$

$$
u_{k j}\left(p_{k j}, q_{k j}\right)=0.5=0.5 p_{k j} q_{k j}, \forall k, j
$$

The market demand is:

$$
\begin{aligned}
& d_{1}(p)=-0.5 p_{11}-p_{21}+2000 \\
& d_{2}(p)=-0.5 p_{21}-p_{22}+2000
\end{aligned}
$$

The manufacturer's production costs are:

$$
\begin{gathered}
f_{1}\left(Q_{3}\right)=\left(\sum_{j=1}^{2} q_{1 j}\right)^{2}+2\left(\sum_{j=1}^{2} q_{1 j}\right)\left(\sum_{j=1}^{2} q_{2 j}\right)+4 \\
f_{2}\left(Q_{3}\right)=2\left(\sum_{j=1}^{2} q_{1 j}\right)^{2}+\left(\sum_{j=1}^{2} q_{1 j}\right)\left(\sum_{j=1}^{2} q_{2 j}\right)+3 \\
Q_{1}: q_{111}=23.3783 \quad q_{112}=23.3783 \quad q_{121}=23.3783 \quad q_{122}=23.3783 \\
q_{211}=31.5861 \quad q_{212}=31.5861 \quad q_{221}=31.5861 \quad q_{222}=31.5861 \\
h_{1}: h_{111}=h_{112}=h_{121}=h_{122}=h_{211}=h_{212}=h_{221}=h_{222}=0 \\
Q_{2}: q_{111}=32.7603 \quad q_{112}=32.7603 \quad q_{121}=21.8643 \quad q_{122}=21.8643 \\
q_{211}=32.7603 \quad q_{212}=32.7603 \quad q_{221}=21.8643 \quad q_{222}=21.8643 \\
h_{2}: h_{111}=h_{112}=h_{121}=h_{122}=h_{211}=h_{212}=h_{221}=h_{222}=1 \\
Q_{3}: q_{11}=0 \quad q_{12}=131.3863 \quad q_{21}=86.7371 \quad q_{22}=0 \\
p: p_{11}=393.6503 \quad p_{12}=393.6065 \quad p_{21}=416.27 \quad p_{22}=393.7633
\end{gathered}
$$

The parameters are set to a: step length $b$, loop verification condition c. Using MATLAB programming, the final

In the distribution of profit, when, that is, the raw material supplier's profit ratio is 0.1 , the manufacturer's profit ratio is
0.3 , and the retailer's profit ratio is 0.7 . it can be found that changes in demand due to emergencies have an impact on the 
entire supply chain. Under the new equilibrium state of the supply chain, demand has increased. The transaction volume of raw material suppliers and manufacturers, manufacturers and retailers, and retailers and the consumer market in the two consumer markets have increased in different proportions. At the same time, the consumer market The transaction price of the product is also higher than when the emergency did not occur.

\section{Summary}

This chapter mainly studies the supply chain network equilibrium and coordination mechanism considering the risk function under emergencies. This chapter takes the supply chain composed of multiple raw material suppliers, multiple manufacturers and multiple retailers as the research object, based on the hyper-network perspective, Use revenue-sharing contracts to build a supply chain super-network coordination model, and apply variational inequalities to analyze the decision-making behaviors of entities at various levels, and explore how decision-makers at various levels can formulate new plans when considering the risk function.

With the further development of the global economy and closer cooperation between enterprises and enterprises, the influence of external factors on the supply chain will also increase. The supply chain balance and coordination under emergencies involved in this article need further research.

In actual enterprise production, the cost of emergencies is not only reflected in the deviation cost, but also involves factors such as the break of the capital chain, the withdrawal of manufacturers, and the serious shortage of raw materials. Under emergencies, these factors appear more or less at the same time. Whether there is any change in the coordination ability and coordination level of the revenue-sharing contract, this needs to be further explored in future research.
None of the models in this article specify the specific industries or product types involved in the supply chain. In actual production activities, products have different characteristics, such as fruits and clothes. Products with different characteristics have different coordination levels of revenue sharing contracts when emergencies occur, and different coordination methods have different coordination levels for the supply chain where the same product is located. The use of different coordination strategies and supply chain emergency research with product characteristics need to be discussed in the right rear research.

\section{References}

[1] Ma Chao, Huang Fuyou, Huang Xiaohui. Revenue-sharing contract with out-of-stock loss based on effort effect and loss aversion. Statistics and Decision. Vol. 36 (2020), No. 10, p. 167-171.

[2] Luo Xinxing, Zhang Peng. Dynamic Supply Chain Revenue Sharing Contract under Variable Promotion Efforts. Journal of Systems Engineering. Vol. 35 (2020), No. 1, p.120-129.

[3] Li Feng, Li Guangxia. Research on benefit distribution of multi-level coal supply chain based on revenue sharing contract Coal Economic Research. Vol. 38 (2018), No. 12, p. 28-38.

[4] Li Yuan, Zhao Daozhi. Supply chain performance considering carbon emission reduction under revenue sharing consignment contract. Chinese Journal of Management Engineering. Vol. 30 (2020), No. 3, p. 188-194.

[5] Wei Guangxing, Xu Guixiao. Analysis of Revenue Sharing Contract Based on Nash Bargaining Fairness Preference. Business Research. Vol. 26 (2016), No. 19, p. 173-185.

[6] Sun Yuling, Hong Meina, Shi Kunran. Revenue-sharing contract of fresh agricultural product supply chain considering fairness concerns. Operations Research and Management. Vol. 24 (2015), No. 6, p. 103-111. 